\title{
Two-proton radioactivity and three-body decay. V. Improved momentum distributions
}

\author{
L. V. Grigorenko, ${ }^{1,2,3,{ }^{*}}$ I. A. Egorova, ${ }^{4}$ M. V. Zhukov, ${ }^{5}$ R. J. Charity, ${ }^{6}$ and K. Miernik ${ }^{7}$ \\ ${ }^{1}$ Flerov Laboratory of Nuclear Reactions, JINR, RU-141980 Dubna, Russia \\ ${ }^{2}$ Gesellschaft für Schwerionenforschung mbH, Planckstrasse 1, D-64291, Darmstadt, Germany \\ ${ }^{3}$ RRC “The Kurchatov Institute," Kurchatov Sq. 1, RU-123182 Moscow, Russia \\ ${ }^{4}$ Bogolubov Laboratory of Theoretical Physics, JINR, RU-141980 Dubna, Russia \\ ${ }^{5}$ Fundamental Physics, Chalmers University of Technology, S-41296 Göteborg, Sweden \\ ${ }^{6}$ Department of Chemistry, Washington University, St. Louis, Missouri 63130, USA \\ ${ }^{7}$ Faculty of Physics, University of Warsaw, PL-00-681 Warsaw, Poland
}

(Received 17 April 2010; published 26 July 2010)

\begin{abstract}
Nowadays quantum-mechanical theory allows one to reliably calculate the processes of $2 p$ radioactivity (true three-body decays) and the corresponding energy and angular correlations up to distances of the order of $10^{3} \mathrm{fm}$. However, the precision of modern experiments has now become sufficient to indicate some deficiency of the predicted theoretical distributions. In this paper we discuss extrapolation along the classical trajectories as a method to improve the convergence of the theoretical energy and angular correlations at very large distances (of the order of atomic distances), where only long-range Coulomb forces are still operating. The precision of this approach is demonstrated using the "exactly" solvable semianalytical models with simplified three-body Hamiltonians. It is also demonstrated that for heavy $2 p$ emitters, the $2 p$ decay momentum distributions can be sensitive to the effect of screening by atomic electrons. We compare theoretical results with available experimental data.
\end{abstract}

DOI: 10.1103/PhysRevC.82.014615

PACS number(s): 21.45.-v, 21.60.Gx, 23.50.+z

\section{INTRODUCTION}

Two-proton radioactivity is the most recently discovered radioactive decay mode of nuclei and it is a very actively developing field. Forty-two years passed between the prediction [1] and the discovery $[2,3]$ of $2 p$ radioactivity, and subsequently, 7 years later, we have several well-studied examples. A number of experiments performed in the last 2-3 years can be characterized as having seminal importance. In particular, correlations in $2 p$ decays have been measured recently in ${ }^{6} \mathrm{Be}[4],{ }^{16} \mathrm{Ne}[5],{ }^{19} \mathrm{Mg}[5,6],{ }^{45} \mathrm{Fe}$ [7], and ${ }^{94} \mathrm{Ag}$ [8], providing qualitatively new information about $2 p$ decays. With correlation information becoming available, $2 p$ decay studies are now becoming a field of research where precise information about structure and continuum dynamics can be obtained. It is clear that our ability to extract useful information from correlations is directly dependent on how well we understand the propagation of particles in the long-range three-body Coulomb field.

From a theoretical point of view, true two-proton decay ( $2 p$ radioactivity) is an exclusively quantum-mechanical phenomenon, which has no analog in classical physics. It is expected to be widely spread along the proton drip line with $Z<50$ owing to peculiarities of the pairing interaction. A consistent quantum-mechanical theory of two-proton radioactivity and "democratic" three-body decays of Coulombic nuclear systems has been developed in the series of papers [9-13] that we continue here and has been applied to different physical cases in Refs. [4] and [14-17]. The complete momentum correlations for the decay of a nonaligned three-body system can be described by two parameters. These parameters are chosen

\footnotetext{
*1grigorenko@yandex.ru
}

in this and our previous studies as the energy distribution parameter $\varepsilon$ between any two of the particles and the angle $\theta_{k}$ between the Jacobi momenta:

$$
\begin{aligned}
\varepsilon & =E_{x} / E_{T}, \quad \cos \left(\theta_{k}\right)=\left(\mathbf{k}_{x} \cdot \mathbf{k}_{y}\right) /\left(k_{x} k_{y}\right), \\
E_{T} & =E_{x}+E_{y}=k_{x}^{2} / 2 M_{x}+k_{y}^{2} / 2 M_{y}, \\
M_{x} & =\frac{A_{1} A_{2}}{A_{1}+A_{2}} M, \quad M_{y}=\frac{\left(A_{1}+A_{2}\right) A_{3}}{A_{1}+A_{2}+A_{3}} M, \\
\mathbf{k}_{x} & =\frac{A_{2} \mathbf{k}_{1}-A_{1} \mathbf{k}_{2}}{A_{1}+A_{2}}, \quad \mathbf{k}_{y}=\frac{A_{3}\left(\mathbf{k}_{1}+\mathbf{k}_{2}\right)-\left(A_{1}+A_{2}\right) \mathbf{k}_{3}}{A_{1}+A_{2}+A_{3}},
\end{aligned}
$$

where $A_{i}$ are the mass numbers of the constituents, $M$ is a nucleon mass, and $E_{T} \equiv Q_{2 p}$ is a two-proton decay energy. For two-proton emitters these parameters can be constructed in two "irreducible" Jacobi systems, called "T" and "Y" (see Fig. 1). A detailed definition of the Jacobi coordinates is given in Ref. [4]. Complete correlation pictures for two-proton decay were, for the first time, calculated in Ref. [11]. Various aspects of the correlations between the decay products have been discussed in the theoretical work in Refs. $[4,5,11,12,14]$.

In ${ }^{6} \mathrm{Be}$ and ${ }^{45} \mathrm{Fe}$, the complete correlation pictures for $2 p$ decay were recently obtained experimentally $[4,7]$. Moreover, the precision of these experimental results is now sufficient to show a deficiency in certain aspects of the predicted momentum distributions in the case of heavy $2 p$ emitters [17]. It was already understood in Ref. [10] that this deficiency is connected to the limited radial range of the calculations and the approximate nature of the boundary conditions employed for the treatment of three-body Coulomb asymptotics.

The classical extrapolation (CE) of momentum distributions was suggested in Ref. [11] as a simple way to estimate the 

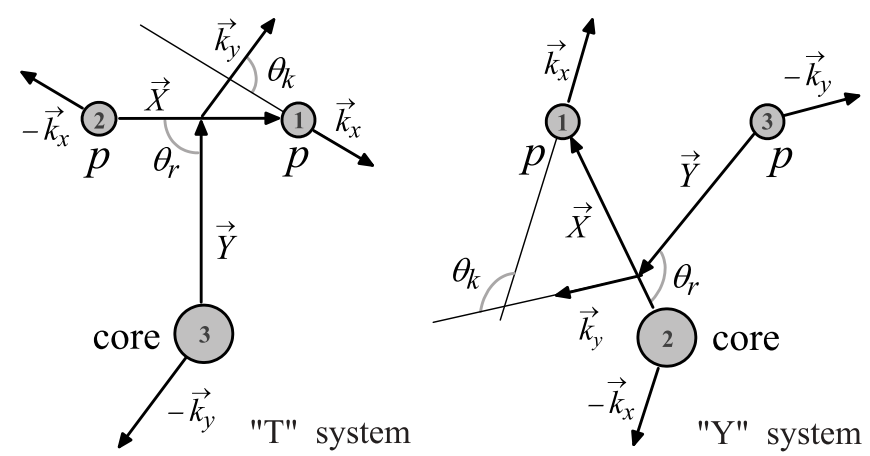

FIG. 1. Independent "T" and "Y" Jacobi systems for the core + $N+N$ three-body system in coordinate and momentum spaces. There are "planar" cases where both the coordinates and the momenta belong to the same plane.

possible influence of the "residual" Coulomb interaction. The basic idea is that, at small distances, particles are propagated by quantum-mechanical equations providing the three-body wave function (WF) $\Psi_{3}^{(+)}$with outgoing asymptotics. At some sufficiently large distance, the WFs are converted into "events" with definite coordinates and momenta by a Monte Carlo (MC) procedure. However, at that time (in 2002 the $2 p$ decay of ${ }^{45} \mathrm{Fe}$ was just discovered, with statistics of the order of 10 events $[2,3]$ ) the need to improve this aspect of our calculations was assigned to the remote future and no detailed studies were performed. Now it seems that the development of the field has reached the stage where the need to improve this aspect of our approach has become evident.

In this work we discuss the method of $\mathrm{CE}$ in detail, demonstrate its reliability by application to exactly solvable three-body models with a simplified Hamiltonian, and consider three "key" cases $\left({ }^{6} \mathrm{Be},{ }^{19} \mathrm{Mg}\right.$, and $\left.{ }^{45} \mathrm{Fe}\right)$ covering a broad range of possible charges, masses, and structures for $2 p$ emitters.

The natural system of units with $\hbar=c=1$ is used in this work.

\section{APPROXIMATE BOUNDARY CONDITIONS}

In this section we sketch the methods used to construct the approximate boundary conditions [10] and outline existing problems. The asymptotic form of the three-body potentials in the hyperspherical harmonics $(\mathrm{HH})$ method is

$V_{K \gamma, K^{\prime} \gamma^{\prime}}(\rho)=\frac{U_{K \gamma, K^{\prime} \gamma^{\prime}}}{\rho^{3+N_{K \gamma, K^{\prime} \gamma^{\prime}}}}+\frac{\mathcal{L}(\mathcal{L}+1)}{\rho^{2}} \delta_{K \gamma, K^{\prime} \gamma^{\prime}}+\frac{v \eta_{K \gamma, K^{\prime} \gamma^{\prime}}}{\rho}$,

where the multi-index $\{K \gamma\}=\left\{K, L, S, l_{x}, l_{y}, s_{x}\right\}$ is a complete set of quantum numbers. The matrix $U_{K \gamma, K^{\prime} \gamma^{\prime}}$ arises owing to contributions from short-range nuclear forces, and $N_{K \gamma, K^{\prime} \gamma^{\prime}} \geqslant 0$ are some integer numbers. The effective contribution of the short-range forces decreases as $\rho^{-3}$ or faster in hypersherical space. The diagonal centrifugal term depends on the "effective angular momentum" $\mathcal{L}=K+3 / 2$. Coulomb pairwise potentials generate the long-range part of the hyperspherical potentials behaving as $\rho^{-1}$. From the technical side, the three-body Coulomb interaction causes problems owing to long-range channel coupling (nonzero nondiagonal "Sommerfeld parameters" $\eta_{K \gamma, K^{\prime} \gamma^{\prime}}$ ) that does not allow one to decouple the $\mathrm{HH}$ equations in the asymptotic region. To deal with this problem, the finite-size potential matrix (in a truncated hyperspherical basis) can be diagonalized with respect to the long-range term by the orthogonal transformation $\tilde{V}=A^{T} V A$ :

$\tilde{V}_{K \gamma, K^{\prime} \gamma^{\prime}}(\rho)=\frac{\tilde{U}_{K \gamma, K^{\prime} \gamma^{\prime}}}{\rho^{3}}+\frac{C_{K \gamma, K^{\prime} \gamma^{\prime}}}{\rho^{2}}+\frac{v \eta_{K \gamma}}{\rho} \delta_{K \gamma, K^{\prime} \gamma^{\prime}}$.

This potential includes nondiagonal "centrifugal" terms $C_{K \gamma, K^{\prime} \gamma^{\prime}}$, and to achieve the asymptotics in the diagonalized representation, we still need to go very far in $\rho$ value, where the terms $\sim \rho^{-2}$ become negligible compared to those $\sim \rho^{-1}$. At such $\rho$ values, the hyper-radial part of the asymptotic solution with a pure outgoing nature can be constructed in the form

$$
\begin{aligned}
\chi_{K \gamma}^{(+)}(\rho) & \sim \sum_{K^{\prime} \gamma^{\prime}} A_{K \gamma, K^{\prime} \gamma^{\prime}}\left[\left(G_{\mathcal{L}_{0}}\left(\eta_{K^{\prime} \gamma^{\prime}}, \rho\right)+i F_{\mathcal{L}_{0}}\left(\eta_{K^{\prime} \gamma^{\prime}}, \rho\right)\right],\right. \\
\Psi_{3}^{(+)} & =\rho^{-5 / 2} \sum_{K \gamma} \chi_{K \gamma}^{(+)}(\rho) \mathcal{J}_{K \gamma}\left(\Omega_{5}\right) .
\end{aligned}
$$

The functions $F$ and $G$ are the ordinary regular and irregular Coulomb functions. HH $\mathcal{J}_{K \gamma}$ are functions of the fivedimensional "solid angle" $\Omega_{5}=\left\{\theta_{\rho}, \Omega_{x}, \Omega_{y}\right\}$. Here $\Omega_{x}$ and $\Omega_{y}$ are ordinary solid angles of the Jacobi vectors $\mathbf{X}$ and $\mathbf{Y}$ [see Eq. (5)] and $\tan \left(\theta_{\rho}\right)=\sqrt{M_{x} / M_{y}} X / Y$. The value $\mathcal{L}_{0}$ should be larger than $3 / 2$ but, otherwise, does not seem to be particularly important. The WFs $\chi^{(+)}$provide the necessary boundary conditions for the decay problem.

The proposed boundary conditions are exact in the truncated hyperspherical basis for a hypersphere of very large radius. However, on a practical level, these two requirements contradict each other: the further movement in radius requires an increase in the basis size; a larger basis size may require a larger radius. Therefore, at some point, the further radial propagation of the solution (with a fixed basis size) leads to a deterioration of its quality. For ${ }^{45} \mathrm{Fe}$ with a decay energy of $1.154 \mathrm{MeV}$ and a basis size of $K_{\max }=20$, radii between 500 and $2000 \mathrm{fm}$ are needed to get reasonable solutions.

There exists an analytical asymptotics of the three-body Coulomb problem (a so-called "Redmond-Merkuriev" asymptotics $[18,19])$, which is presumably applicable to the true three-body decay. Practical application of this asymptotics is technically complicated and it seems that very limited experience in using such an asymptotics exists. For the moment we are going to avoid these complexities and to demonstrate that there exists a simple and practical way to treat the problem.

\section{EXTRAPOLATION ALONG CLASSICAL TRAJECTORIES}

To perform a CE of the quantum-mechanical result, we need to switch from a WF to classical trajectories. This should be done at some closed surface around the decay region. The procedure becomes especially simple if the whole surface is located in the region of classically allowed motion. Then the 
flux vectors at the surface can provide initial conditions for classical trajectories.

When using the $\mathrm{HH}$ coordinates there is only one variable $\rho$, which has a dimension of length [the six-dimensional flux can be calculated for different $\rho$ values; see Eq. (8)]. Therefore, it is natural in this approach to select a hypersphere with a large radius $\rho_{\max }$ as such a surface. We see later that tiny regions on a hypersphere with a large radius where the pairwise distances appear to be small do not lead to problems, as the WFs in these regions are strongly suppressed. This happens because of the energy conditions defining the true $2 p$ decay: there are no long-living states in either pair of the three constituents, and the strong Coulomb repulsion rapidly "expels" particles from the regions where they are close to each other.

A less evident, but important requirement is that the hyperradius $\rho_{\max }$ is large enough that the typical distances between each pair of particles significantly exceeds the typical quantum coherence length (the "corpuscular" aspect of the problem then far prevails over the possible wave effects). This is a complicated issue, and in each case an acceptable minimal value of $\rho_{\max }$ should be defined by numerical experiment.

The classical trajectories formed at this hypersphere $\rho_{\max }$ are propagated to distances $\rho_{\text {ext }} \gg \rho_{\max }$ at which the momentum distributions are stabilized (what this means exactly we see later). After this, the momentum distributions are reconstructed from the set of trajectories.

The pairwise distances, the Jacobi vectors, and the hyperradius are connected by the following relations:

$$
\begin{aligned}
\mathbf{r}_{12} & =\mathbf{X}, \quad \mathbf{r}_{23}=\mathbf{Y}-c_{1} \mathbf{X}, \quad \mathbf{r}_{31}=\mathbf{Y}+c_{2} \mathbf{X}, \\
\rho^{2} & =\frac{A_{1} A_{2}}{A_{1}+A_{2}} X^{2}+\frac{\left(A_{1}+A_{2}\right) A_{3}}{A_{1}+A_{2}+A_{3}} Y^{2}, \\
c_{1} & =A_{1} /\left(A_{1}+A_{2}\right), \quad c_{2}=A_{2} /\left(A_{1}+A_{2}\right) .
\end{aligned}
$$

In the definition of the hyper-radius $\rho$, particle $A_{3}$ should be a heavy core if $X$ and $Y$ are defined in the "T" Jacobi system, and either $A_{1}$ or $A_{2}$ should be a core in the "Y" Jacobi system (see also Fig. 1 for the numbering convention).

Newton equations of motion for the Jacobi vectors are used to avoid the extra degrees of freedom connected with the center-of-mass motion:

$$
\begin{aligned}
& M_{x} \ddot{\mathbf{X}}=\frac{\alpha Z_{1} Z_{2} \mathbf{X}}{X^{3}}-\frac{\alpha Z_{2} Z_{3} c_{1} \mathbf{r}_{23}}{r_{23}^{3}}+\frac{\alpha Z_{3} Z_{1} c_{2} \mathbf{r}_{31}}{r_{31}^{3}}, \\
& M_{y} \ddot{\mathbf{Y}}=\frac{\alpha Z_{2} Z_{3} \mathbf{r}_{23}}{r_{23}^{3}}+\frac{\alpha Z_{3} Z_{1} \mathbf{r}_{31}}{r_{31}^{3}} .
\end{aligned}
$$

The particular choice of the form of Eqs. (6) ("T" or "Y" Jacobi system) and the numerical precision in solving this system are not practical obstacles for getting the correct classical trajectories.

The initial conditions for these equations are defined on the hypersphere of the maximal radius achieved in the quantummechanical calculations:

$$
\begin{aligned}
\left\{\rho_{\max }, \Omega_{\rho}^{(r)}\right\} & \rightarrow\{\mathbf{X}(0), \mathbf{Y}(0)\}, \\
\left\{\mathbf{j}_{x}\left(\rho_{\max }, \Omega_{\rho}^{(r)}\right), \mathbf{j}_{y}\left(\rho_{\max }, \Omega_{\rho}^{(r)}\right)\right\} & \rightarrow\{\dot{\mathbf{X}}(0), \dot{\mathbf{Y}}(0)\},
\end{aligned}
$$

where $\Omega_{\rho}^{(r)}$ is a randomly generated five-dimensional hyperangle selected by the MC procedure according to the WF density
$\left|\Psi_{3}^{(+)}\right|^{2}$ at $\rho=\rho_{\max }$. The flux associated with the Jacobi vectors is defined in an ordinary way:

$$
\mathbf{j}_{i}\left(\rho, \Omega_{\rho}\right)=\frac{1}{M_{i}} \operatorname{Im}\left[\Psi_{3}^{(+) \dagger} \nabla_{i} \Psi_{3}^{(+)}\right] .
$$

In the quantum-mechanical model of three-body decays [9-13], the total flux $j$ through the hypersphere $\rho=\rho_{\max }$ defines the width

$$
\Gamma=j / N
$$

where $N$ is the normalization of the $\mathrm{WF} \Psi_{3}^{(+)}$in the internal region. The momentum distribution (density distribution) is found as the derivative of the flux $d j /\left[d \varepsilon d \cos \left(\theta_{k}\right)\right]$; see Eq. (1). In this work we compare the quantum-mechanical distributions calculated at $\rho=\rho_{\max }$ (henceforth called "without CE" or "initial") with distributions obtained by $\mathrm{CE}$ to $\rho=\rho_{\text {ext }}$ (henceforth, "with CE” or "final”).

\section{A. Treatment of spins}

It is implied in the preceding section that the flux is averaged over the initial spin states and summed over the final spin states. Therefore the components of the $\mathrm{WF}_{3}^{(+)}$with different total spin $S$ can be considered different "particles" whose contributions to the total momentum distribution should be added incoherently.

In general, three particles (or two Jacobi vectors) define a plane. Within this plane, the set of six equations (6) can be reduced to four equations. However, the momentum vectors do not necessarily belong to this plane. It is evident that the geometry of the problem remains planar in the case of zero angular momenta of the $X$ and $Y$ subsystems (this situation is shown in Fig. 1). For nonzero angular momenta, some additional considerations are required.

Let us consider the flux field induced by the ordinary two-body WF with $l \neq 0$. For $m=0$, the flux is purely radial as the angular part of the WF $Y_{l m}$ is real (flux is an imaginary part of the gradient matrix element). For purely radial flux, the classical angular momentum associated with the particular trajectory is 0 (radius and momentum vectors are collinear). This can be seen as a source of confusion, as the quantum-mechanical momentum of the WF and the classical momentum of the selected trajectory are explicitly different. The answer seems to be that the classical characteristic of the trajectory should be related to the average corresponding to the characteristic of the WF.

In the three-body case, the ground-state (g.s.) WFs typically have two major components: the dominating $L=0$ component and an "admixture" $L=1$ component. We imply here that a spin- 0 core is considered; the two spin- $1 / 2$ protons can then be coupled into the total spin $S=0$ or $S=1$. The $L=0$ component of the WF is formed by terms with angular momenta in the subsystem $l_{x}=l_{y}$. It is easy to check that the angular part of this WF $\left[Y_{l_{x}} \otimes Y_{l_{x}}\right]_{00}$ is real and thus the classical angular momentum associated with any trajectory induced by this WF is 0 . The decay in this case is planar (we mean that for any generated event, a plane can be selected in which the coordinate vectors and the momentum vectors of all three particles are simultaneously located). 
It is more complicated when the $L=1$ component is considered. It is possible to demonstrate that for the $\left[Y_{l_{x}} \otimes\right.$ $\left.Y_{l_{x}}\right]_{1 M}$ component of the WF with $M=0$, the configuration of the classical momenta is planar, while for $M \neq 0$, the planes formed by the three radii and by the three momenta do not coincide. However, according to the Wigner-Eckart theorem, to define the observables it is sufficient to calculate the matrix elements for only one projection and the rest are reconstructed by the angular momentum algebra. Therefore, it seems sufficient to calculate the distributions for $M=0$ (planar case calculations are especially simple), while the distributions for $M= \pm 1$ should be the same.

\section{TEST CASES OF SOLVABLE SEMIANALYTICAL MODELS}

In Ref. [12], a semianalytical model was developed that allows one to treat exactly the asymptotic behavior of the three-body Coulomb WF for certain simplified three-body Hamiltonians. The basic idea of the model is that instead of the real three-body Hamiltonian,

$$
H_{3}=T_{x}+T_{y}+V_{12}\left(\mathbf{r}_{12}\right)+V_{23}\left(\mathbf{r}_{23}\right)+V_{31}\left(\mathbf{r}_{31}\right),
$$

we use the model Hamiltonian depending not on pairwise vectors $\mathbf{r}_{i j}$ but on the Jacobi vectors $\mathbf{X}$ and $\mathbf{Y}$ :

$$
H_{3}=T_{x}+T_{y}+V_{x}(\mathbf{X})+V_{y}(\mathbf{Y})+V_{3}(\rho) .
$$

The three-body potential $V_{3}(\rho)$ used in this work has the Woods-Saxon form

$$
\begin{aligned}
V_{3}(\rho) & =V_{3}^{0}\left(1+\exp \left[\left(\rho-\rho_{0}\right) / a_{\rho}\right]\right)^{-1}, \\
\rho_{0} & =\sqrt{2} 1.2\left(A_{\text {core }}+1\right)^{1 / 3},
\end{aligned}
$$

with a small value of the diffuseness parameter $a_{\rho}=0.4 \mathrm{fm}$. The depth $V_{3}^{0}$ of this potential is used to control the decay energy of the system. The potentials $V_{x}$ and $V_{y}$ contain the nuclear and the Coulomb contributions. The Coulomb potential of the homogeneously charged sphere with a radius $r_{\mathrm{sph}}$ is used. The nuclear parts are described by Woods-Saxon form factors, with radii taken from systematics.

In conjunction with this simplified Hamiltonian of Eq. (11), we can introduce an auxiliary Hamiltonian,

$$
\bar{H}_{3}=T_{x}+T_{y}+V_{x}\left(\mathbf{X}_{12}\right)+V_{y}\left(\mathbf{Y}_{23}\right),
$$

for which the Green's function can be constructed in analytical form,

$$
G_{E_{T}}^{(+)}\left(\mathbf{X Y}, \mathbf{X}^{\prime} \mathbf{Y}^{\prime}\right)=\frac{1}{2 \pi i} \int_{-\infty}^{\infty} d E_{x} G_{E_{x}}^{(+)}\left(\mathbf{X}, \mathbf{X}^{\prime}\right) G_{E_{y}}^{(+)}\left(\mathbf{Y}, \mathbf{Y}^{\prime}\right)
$$

where $E_{T}$ is the total decay energy, and $E_{x}=\varepsilon E_{T}$ and $E_{y}=$ $(1-\varepsilon) E_{T}$ are the energies of the Jacobi subsystems. These two-body Green's functions correspond to the $X$ and $Y$ subHamiltonians of $\bar{H}_{3}$. Based on Eq. (15), the width and the energy distribution for the system defined by the Hamiltonian of Eq. (11) can be obtained from

$$
\frac{d \Gamma}{d \varepsilon}=\frac{d j}{d \varepsilon}=\frac{8}{\pi} E_{T} \frac{M_{x} M_{y}}{k_{x}(\varepsilon) k_{y}(\varepsilon)}|A(\varepsilon)|^{2},
$$

where $d j / d \varepsilon$ is a derivative of the flux in the asymptotic region. For a particular set of quantum numbers $l_{x}, l_{y}$, the amplitudes
$A(\varepsilon)$ are defined via the scattering eigenfunctions $\varphi_{l_{i}}$ of subHamiltonians of (14):

$$
\begin{aligned}
A(\varepsilon)= & \int_{0}^{\infty} d X \int_{0}^{\infty} d Y \varphi_{l_{x}}\left(k_{x}(\varepsilon) X\right) \varphi_{l_{y}}\left(k_{y}(\varepsilon) Y\right) \\
& \times V_{3}(\rho) \varphi_{L l_{x} l_{y} S}(X, Y) .
\end{aligned}
$$

The WF $\varphi_{L l_{x} l_{y} S}(X, Y)$ is the quasi-stationary eigenfunction of (11), deduced in a three-body hyperspherical approach. The particular choice of the boundary conditions for this WF (for a sufficiently large radius of the "box") is not important in the model. The quasi-stationary WF is normalized to unity in the internal region, which gives the identity $d \Gamma / d \varepsilon \equiv d j / d \varepsilon$ in Eq. (16).

The results obtained in this model are quoted here as "exact," as they do not suffer from any convergence/stability issues. In Secs. IV A and IV B, we use models with different simplified Hamiltonians to test the CE procedure in the case of the ${ }^{19} \mathrm{Mg}$ g.s. decay, and only after that do we turn to more realistic situations.

\section{A. Direct-decay model}

The Hamiltonian of Eq. (11) constructed in the "Y" Jacobi system corresponds to some physically well-justified approximations: (i) we neglect the proton-proton interaction and (ii) for one of the core-proton potentials we use the Jacobi $Y$ variable instead of the relative distance. The latter assumption becomes correct in the limit of an infinitely heavy core and thus should work well for heavy $2 p$ emitters.

Let us consider the "Y" system where the subsystem \{core + proton is taken as an effective particle lying on the $X$ coordinate as shown in Fig. 1:

$$
V^{\text {coul }}=\frac{\alpha Z_{1} Z_{2}}{X}+\frac{\alpha\left(Z_{1}+Z_{2}\right) Z_{3}}{Y} .
$$

In this case, we include both pairwise interactions, $V_{x}^{\text {nucl }}$ and $V_{y}^{\text {nucl }}$. A system with such a composition of potentials in the "Y" system was labeled "two final-state interactions" in Ref. [12].

For the ${ }^{19} \mathrm{Mg}$ g.s., we assume the pure $d$-wave structure $l_{x}=l_{y}=2$ in this model. The nuclear Woods-Saxon potential was used, with radius

$$
r_{0}=1.2(A+1)^{1 / 3}
$$

and diffuseness $a=0.65 \mathrm{fm}$. The depth of the potentials was adjusted to give an energy of $1.3 \mathrm{MeV}$ for the g.s. resonance in ${ }^{18} \mathrm{Na}$ [6], and the Coulomb potential of the charged sphere with radius

$$
r_{\mathrm{sph}}=\sqrt{\frac{5}{3}\left(1.2 A^{1 / 3}\right)^{2}+0.8^{2}}
$$

was used. In these expressions, one should substitute $A=A_{2}$ in the $X$ subsystem and $A=A_{2}+1$ in the $Y$ subsystem. In this model, we obtained a half-life of $T_{1 / 2}=58$ ps (corresponding to $\Gamma=7.9 \times 10^{-11} \mathrm{MeV}$ ), which is in qualitative agreement with the experimental value for ${ }^{19} \mathrm{Mg}\left(T_{1 / 2}=4 \mathrm{ps}[6]\right)$.

The radial convergence of the energy distribution $\varepsilon$ in this model for some classical trajectories is illustrated in Fig. 2. The trend of the $\mathrm{CE}$ is to make the energy distribution narrower. 

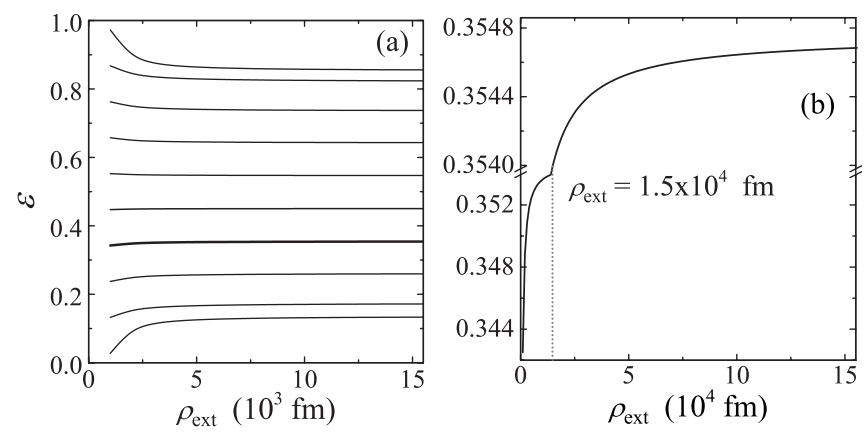

FIG. 2. (a) Classical trajectories for ${ }^{19} \mathrm{Mg}$ in a direct decay model $\left(\rho_{\max }=1000 \mathrm{fm}, E_{T}=0.75 \mathrm{MeV}\right)$. (b) One selected trajectory on a large scale; the dotted vertical line corresponds to the scale of (a).

The visual stability of the distributions is achieved at distances of about $\rho_{\text {ext }} \sim 7000 \mathrm{fm}$ [Fig. 2(a)]. On a larger scale, a certain drift of the trajectories can be seen up to much larger distances [Fig. 2(b)].

The effect of the CE on the energy distributions is demonstrated in Fig. 3. The energy distributions have a characteristic bell shape. Figure 3(a) shows the energy distributions calculated with the quantum-mechanical three-body model [12] for different $\rho_{\max }$ values. The calculated result tends toward the "exact" result of Eq. (16), shown by the solid light (gray) curve. However, this convergence is very slow and some discrepancy remains even for the largest available $\rho_{\max }$. Figure 3(b) shows the distributions obtained with the $\mathrm{CE}$. These distributions are clearly wrong for $\rho_{\max } \lesssim 500 \mathrm{fm}$. However, for larger $\rho_{\max }$, they stabilize and reproduce the results of the solvable model [Eq. (16)] within the width of the curve.

\section{B. "Diproton" model}

The word "diproton" in quotation marks is the name of this model, as it is different from the diproton model typically used in the literature. The diproton correlation in our model is not

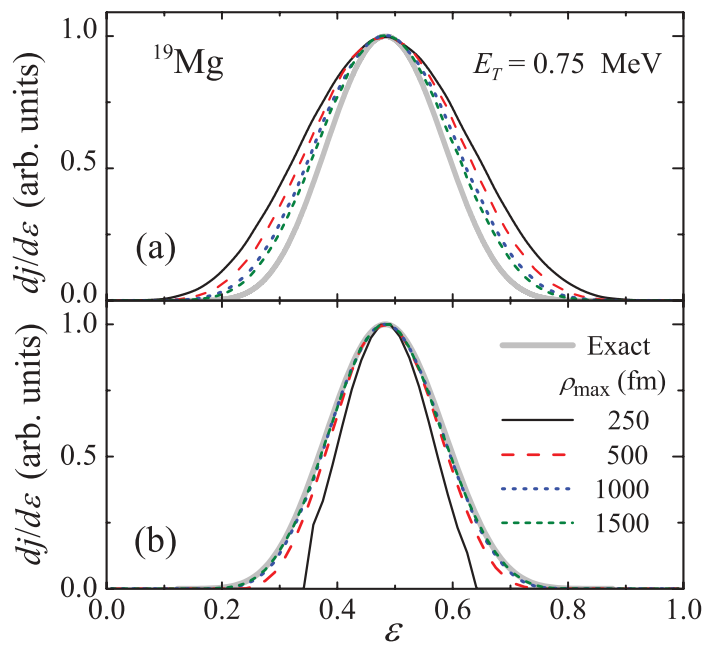

FIG. 3. (Color online) Energy distribution for ${ }^{19} \mathrm{Mg}$ with different $\rho_{\max }$ values without (a) and with (b) classical extrapolation in the direct decay model. Calculations were performed with $E_{\mathrm{T}}=$ $0.75 \mathrm{MeV}, K_{\max }=20$, and (b) with $\rho_{\text {ext }}=40000 \mathrm{fm}$. The solid light (gray) curve shows the exact result of Eq. (16) (same in both plots). introduced statically (which means "by hand") but is treated dynamically. In Ref. [12], we have demonstrated that when introduced appropriately for configurations with the lowest possible angular momenta in the subsystems, the diproton model can provide only a very small value for the $2 p$ width. For decays of higher- $l$ configurations, like $\left[p^{2}\right]$ and $\left[d^{2}\right]$ for $0^{+}$states, this model overestimates the width. Therefore, it is not applicable in practice, in contrast to the widespread belief.

In this work we apply the diproton model, not for realistic estimates, but for testing purposes. The diproton model gives very sharp energy distributions focused at low $p$ - $p$ energies. So we use it to determine whether the CE procedure works for conditions of strong kinematical focusing.

In the diproton model, Eqs. (11)-(17) are used in the "T" system, where the core $\left\{A_{3}, Z_{3}\right\}$ interacts with the two protons as if they were an effective particle $\left\{A_{1}+A_{2}, Z_{1}+Z_{2}\right\}$. The Coulomb potential of the simplified Hamiltonian can be written in the form

$$
V^{\text {coul }}=\frac{\alpha Z_{1} Z_{2}}{X}+\frac{\alpha\left(Z_{1}+Z_{2}\right) Z_{\text {core }}}{Y} .
$$

Note that this is a model with only one nuclear pairwise interaction $V_{x}^{\text {nuc }}(X)$ in the $p$ - $p$ channel (the second interaction can be put to 0 ), and therefore the model is called 'one final-state interaction" in Ref. [12]. The proton-proton nuclear potential for an $s$ wave is taken as a single Gaussian,

$$
V(r)=V_{0} \exp \left[-\left(r / r_{0}\right)^{2}\right],
$$

with $V_{0}=-31 \mathrm{MeV}$ and $r_{0}=1.8 \mathrm{fm}$ reproducing the lowenergy $s=0$ nucleon-nucleon phase shifts. The Coulomb potential of the charged sphere with radius

$$
r_{\mathrm{sph}}=\sqrt{\frac{5}{3}\left(1.2 A_{\text {core }}^{1 / 3}\right)^{2}+\frac{5}{3}\left(1.2 \times 2^{1 / 3}\right)^{2}}
$$

is used in the $Y$ coordinate. The half-life of ${ }^{19} \mathrm{Mg}$ obtained in this model is $T_{1 / 2}=0.39 \mathrm{ps}$ (corresponding to $\Gamma=1.2 \times$ $\left.10^{-9} \mathrm{MeV}\right)$.

The radial convergence of the energy $\varepsilon$ in this model for some classical trajectories is illustrated in Fig. 4. The trend of the $\mathrm{CE}$ is for the trajectories to drift toward the more narrow "diproton" peak in the energy spectrum. The convergence trend is analogous to the direct-decay model, with several thousand femtometers required for reasonable stabilization and more
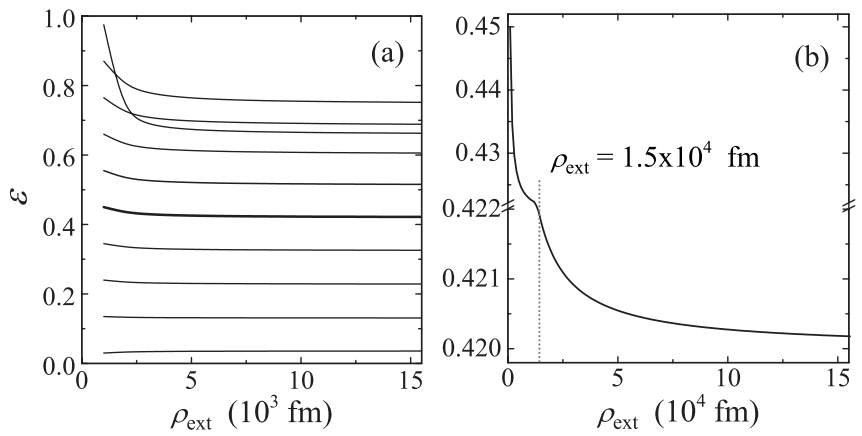

FIG. 4. (a) Classical trajectories for ${ }^{19} \mathrm{Mg}$ in the "diproton" model $\left(\rho_{\max }=1000 \mathrm{fm}, E_{T}=0.75 \mathrm{MeV}\right)$. (b) One selected trajectory on a large scale; the dotted vertical line corresponds to the scale of (a). 


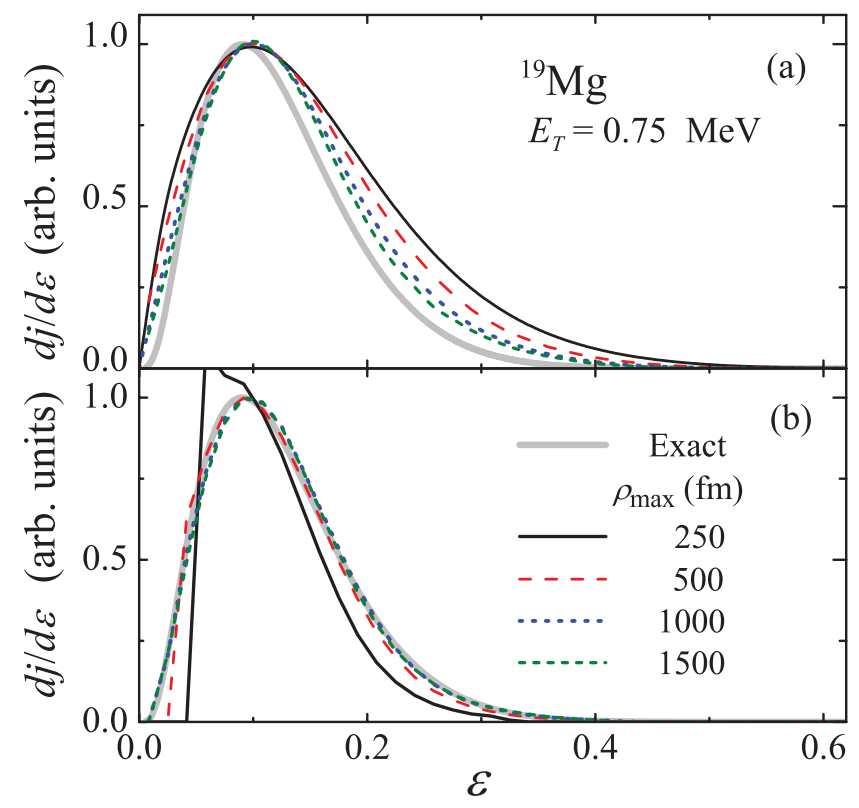

FIG. 5. (Color online) Energy distributions in ${ }^{19} \mathrm{Mg}$ for the "diproton" model without (a) and with (b) classical extrapolation. Calculation results are shown for different $\rho_{\max }$ values. Calculations were performed with $E_{T}=0.75 \mathrm{MeV}, K_{\max }=14$, and (b) with $\rho_{\text {ext }}=10^{5} \mathrm{fm}$. Solid light (gray) curves show the "exact" result of Eq. (16) (the same for both plots).

than a hundred thousand femtometers required for complete stability.

The effect of the CE on the energy distribution is demonstrated in Fig. 5. The case appears to be completely analogous to the direct-decay model. Figure 5(a) shows the energy distributions calculated within our three-body hyperspherical quantum-mechanical approach for different $\rho_{\max }$ values. The quantum-mechanical results tend toward the "exact" result, Eq. (16), but only very slowly. The distributions provided by the CE [see Fig. 5(b)] contain artifacts for $\rho_{\max } \lesssim 500 \mathrm{fm}$, but for larger $\rho_{\max }$ they stabilize and reproduce the result of the solvable model, Eq. (16), within the width of the curve.

\section{Brief conclusions}

Before we continue studies of realistic cases, let us outline what we can conclude on the basis of the exactly solvable models with simplified Hamiltonians.

(i) The quantum-mechanical calculations performed for $\rho_{\max }$ of a few thousand femtometers give energy distributions that have visible deviations from the "exact" results obtained in the semianalytical model. The extrapolated distributions practically coincide with the exact ones.

(ii) The CE provides decent results only if the starting point for the extrapolation is sufficiently large. Pragmatically, this means that the classical trajectories in the kinematical space $\left\{\varepsilon, \cos \left(\theta_{k}\right)\right\}$ should be quite short. The same should be true in the conjugated coordinate space. It can be expected that the criterion of a successful transition from quantum to classical calculation is that the classical variation of a position in some space should be smaller than the corresponding coherence length.

(iii) Distances of tens of thousands of femtometers are needed to achieve complete stabilization of classical trajectories in practice. Some very minor drift continues after that, reflecting the long-range nature of the Coulomb interaction. However, it is evident that distances of $\sim 10^{5} \mathrm{fm}$ are already atomic-scale distances and the nuclear Coulomb effects should be suppressed for larger distances, owing to some form of electron screening.

Near-perfect convergence of the extrapolated distributions to those calculated in the exact semianalytical models with the simplified Hamiltonians is not a proof that the procedure should work perfectly in the case of a complete three-body Hamiltonian. However, it is very encouraging and we can expect that the quality of convergence in the realistic case will be very similar, as the kinematical conditions for the decay in the simplified models are chosen to be the same as in the realistic cases.

\section{REALISTIC THREE-BODY CASES}

For the models with simplified Hamiltonians, we demonstrated only the energy distributions (angular distributions are trivial), and only in one Jacobi system (the one in which the particular semianalytical model is formulated). Conversion of the distribution into the other Jacobi system in this case does not provide additional information. For realistic calculations we demonstrate complete correlation pictures (on the kinematical $\left\{\varepsilon, \cos \left(\theta_{k}\right)\right\}$ plane) simultaneously in both the " $T$ " and the "Y" Jacobi systems. It should be understood that correlation pictures in the "T" and "Y" Jacobi systems are just different representations of the same physical phenomenon. Conversion between these distributions is trivial. Nevertheless, we systematically demonstrate both of them simultaneously, as each representation allows us to reveal different aspects of the correlations (e.g., see Ref. [17]).

\section{A. Decay of ${ }^{6} \mathbf{B e}$}

Very precise complete correlation data were recently obtained for ${ }^{6} \mathrm{Be}$ in Ref. [4]. Detailed theoretical studies of $2 p$ decay of the ${ }^{6} \mathrm{Be} 0^{+}$g.s. were carried out in that work and compared to the experimental data. The dynamical range of about $\rho_{\max }=1000 \mathrm{fm}$ used in these calculations was estimated in Ref. [4] to be sufficient for essentially complete convergence of the momentum distributions. A very nice agreement between theory and experiment was found in this work. We would like to check here whether the conclusions reached in Ref. [4] can be influenced by a more careful treatment of the momentum distributions.

The classical trajectories for ${ }^{6} \mathrm{Be}$ in the kinematical space are all very short. Only trajectories corresponding to small initial interparticle distances $\left[\varepsilon \sim 0.5, \cos \left(\theta_{k}\right) \sim \pm 1\right.$ in the "T" system] have noticeable lengths. The complete correlation densities without and with extrapolation are shown in Fig. 6 (this is the calculation with potential set P2 from Ref. [4], which was found to be the optimal choice in that work). The 

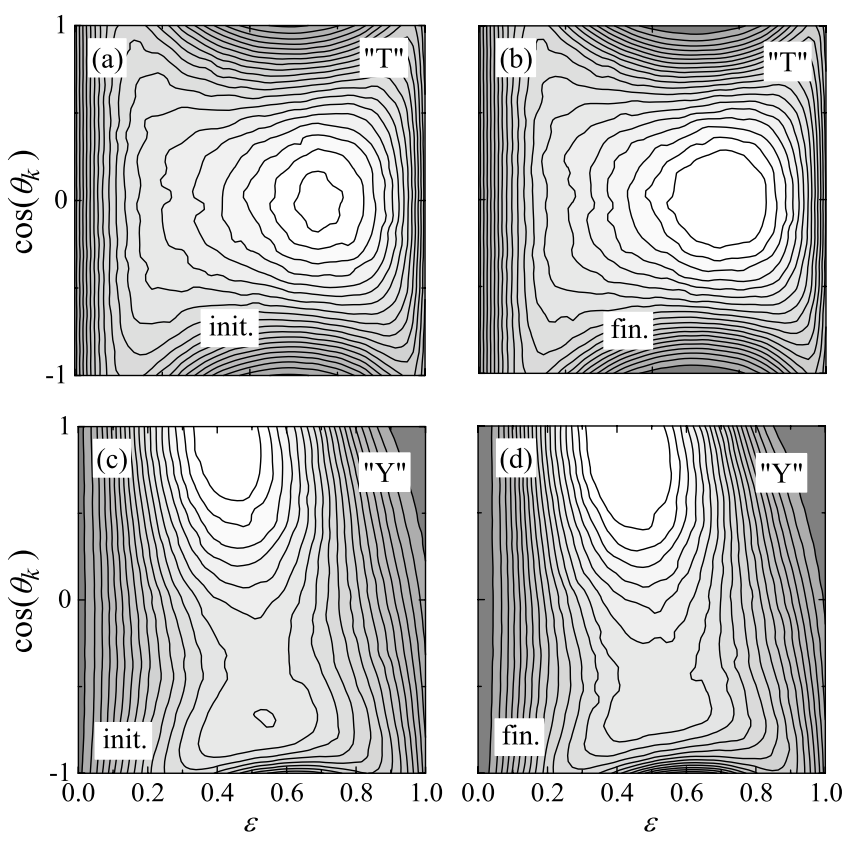

FIG. 6. Contour maps of the momentum density distribution on the kinematical plane $\left\{\varepsilon, \cos \left(\theta_{k}\right)\right\}$ for ${ }^{6} \mathrm{Be}$ in the "T" ( $\left.\mathrm{a}, \mathrm{b}\right)$ and "Y" (c, d) Jacobi coordinate systems without (a, c; "init.") and with (b, d; "fin.") classical extrapolation.

distributions are very similar except for the aforementioned regions of small initial interparticle distances. A closer look at these regions is provided in the inclusive distributions in Fig. 7.
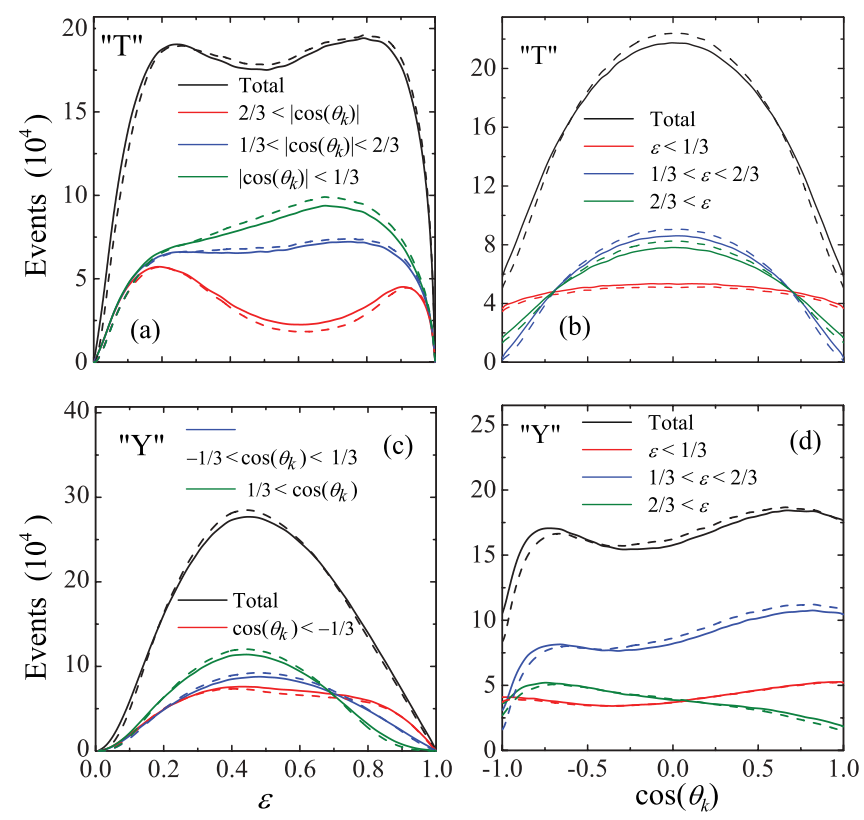

FIG. 7. (Color online) Inclusive energy and angular distributions for ${ }^{6} \mathrm{Be}$ in " $\mathrm{T}$ " (a, b) and "Y" (c, d) Jacobi coordinate systems without (solid curves) and with (dashed curves) classical extrapolation. Darkest (black) curves show the total distribution and color-coded lines show the inclusive distributions for certain energy and angular bins (described in the keys).
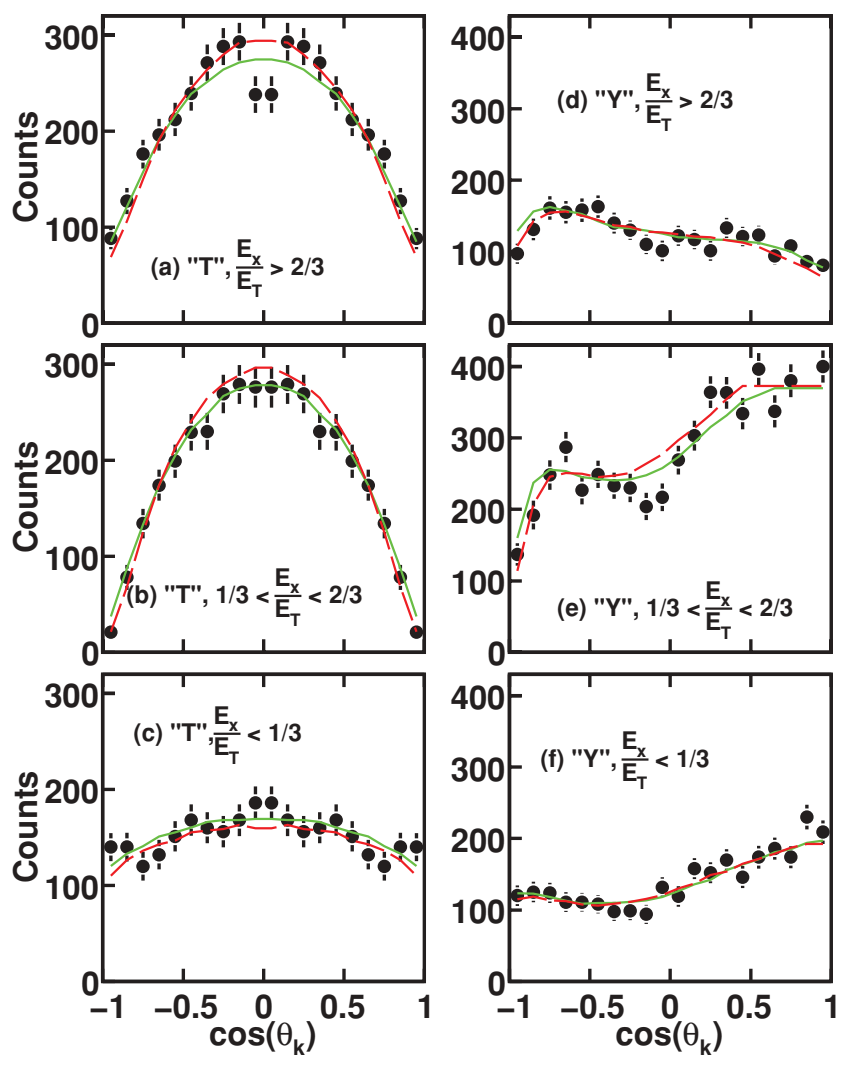

FIG. 8. (Color online) Comparison of experimental (data points [4]) and predicted (curves) $\cos \left(\theta_{k}\right)$ distributions in the " $\mathrm{T}$ " $(\mathrm{a}, \mathrm{c})$ and "Y" (b, d) Jacobi systems for the indicated gates on the $\varepsilon=E_{x} / E_{T}$ parameter. The solid (green) and dashed (red) curves correspond to the three-body calculations without and with classical extrapolation. The effect of the detector bias and resolution is included.

The maximal effect is found at small $\varepsilon$ values (corresponding to the lowest relative-energy motion between two of the particles) or for an angular distribution in the middle energy bins around $\cos \left(\theta_{k}\right) \sim \pm 1$ (in the " $\mathrm{T}$ " system) and $\cos \left(\theta_{k}\right) \sim-1$ (in the "Y" system).

Comparisons with experimental angular distributions [4] are shown in Fig. 8. The theoretical curves here are visibly distorted (relative to Fig. 7), as the comparison is based on the full MC simulation of the experimental setup [4], which takes into account the effects of the experimental bias and resolution. The effect of the CE is at the limit of the experimental sensitivity. Quantitatively the $\chi^{2} / v$ values without extrapolation are 1.17 (in the " $\mathrm{T}$ " system) and 1.14 (in the "Y" system). The same values with extrapolation are found to be 1.20 and 1.16, respectively. This is a little worse, but not really significant. In contrast, there seems to be a minor improvement of the agreement for the parts of the middle energy bins mentioned in the previous paragraph.

The properties of the ${ }^{6} \mathrm{Be}$ continuum are now being actively investigated. New higher precision experiments were performed recently at NSCL (Michigan State University) and at Flerov Laboratory (JINR, Dubna, Russia). The expected precision of these experiments would make the improvement 


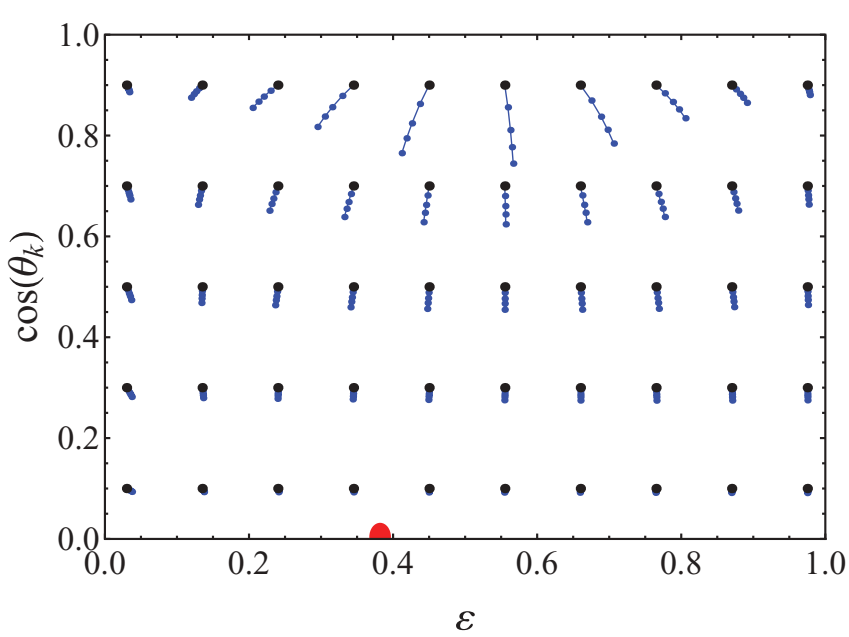

FIG. 9. (Color online) Classical trajectories on the kinematical plane $\left\{\varepsilon, \cos \left(\theta_{k}\right)\right\}$ for ${ }^{19} \mathrm{Mg}$ in the "T" Jacobi system. Starting points (larger circles) correspond to $\rho_{\max }=1000 \mathrm{fm}$. Dots in the curves correspond to $\rho_{\mathrm{ext}}=1300,2000,3500$, and $10^{5} \mathrm{fm}$. The (red) circle on the $x$ axis, $\cos \left(\theta_{k}\right)=0$, corresponds to a stationary point; see the discussion in Sec. VIC.

of the theoretical distributions introduced in this work a necessary part of the data interpretation.

\section{B. Decay of ${ }^{19} \mathrm{Mg}$}

A systematic view of the classical trajectories on the kinematical plane for ${ }^{19} \mathrm{Mg}$ is given in Fig. 9. The "lengths" of the trajectories here are significant: typically about $10 \%$ $15 \%$ of the kinematical variable range, thus making the $\mathrm{CE}$ procedure necessary for quantitative calculations of the momentum distributions.

An improvement of the momentum distribution owing to $\mathrm{CE}$ is demonstrated in Fig. 10 for the complete momentum distributions and in Fig. 11 for the inclusive ones. It can be seen that the angular distributions in the $\mathrm{T}$ and the energy distributions in the "Y" Jacobi systems are the most sensitive to the extrapolation. The effect of the extrapolation on the distributions in certain energy and angular bins can be very large. The energy distribution in the " $T$ " system is only slightly modified by the $\mathrm{CE}$, but it is interesting to note that for very small $\varepsilon$ values (where the $p$ - $p$ Coulomb interaction is expected to be most active), the extrapolated distribution is visibly suppressed.

Unfortunately the available experimental data on the momentum distributions in ${ }^{19} \mathrm{Mg}$ [6] do not provide complete distributions but provide distributions projected on a plane (perpendicular to the incident beam axis). Such distributions integrated over one variable have lost some information and can be more complicated to interpret.

\section{Decay of ${ }^{45} \mathrm{Fe}$}

The ${ }^{45} \mathrm{Fe}$ nucleus is the heaviest $2 p$ emitter studied so far and the effect of the CE is the largest (see Fig. 12).

Radial stabilization of the values $\varepsilon$ and $\cos \left(\theta_{k}\right)$ for one selected trajectory is demonstrated in Fig. 13 (this trajectory is shown in the light gray ellipse in Fig. 12). The trajectories are well "converged" by about (3-4) $\times 10^{4} \mathrm{fm}$ but some drift
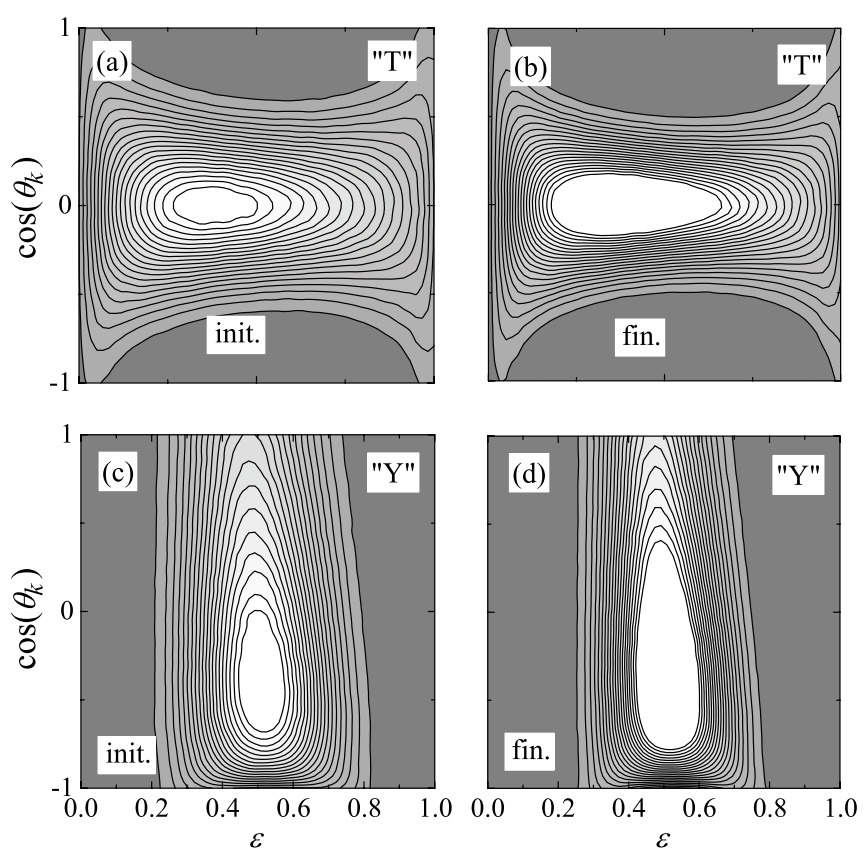

FIG. 10. Contour maps of the momentum density distribution on the kinematical plane $\left\{\varepsilon, \cos \left(\theta_{k}\right)\right\}$ for ${ }^{19} \mathrm{Mg}$ in the " $\mathrm{T}$ " $(\mathrm{a}, \mathrm{b})$ and " $\mathrm{Y}$ " (c, d) Jacobi coordinate systems without (a, c; "init.") and with (b, d; "fin.") classical extrapolation.

continues up to much larger $\rho$ values. In real experimental situations, this slow drift can be suppressed by electron screening, which is discussed separately in Sec. VIB.
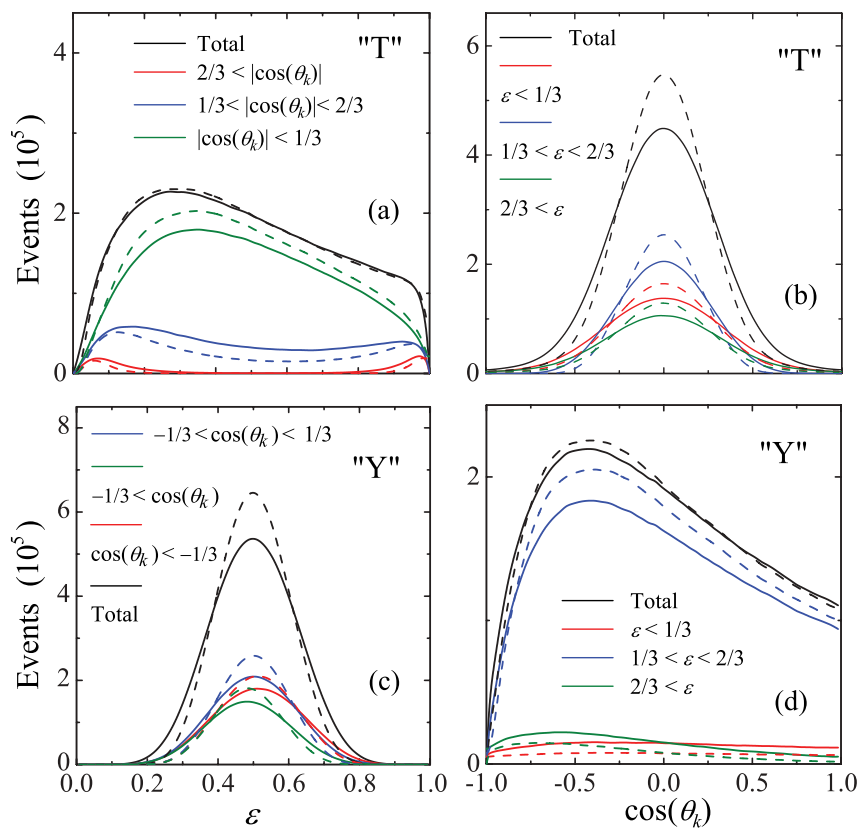

FIG. 11. (Color online) Inclusive energy and angular distributions for ${ }^{19} \mathrm{Mg}$ in the "T" (a, b) and "Y" (c, d) Jacobi coordinate systems without (solid curves) and with (dashed curves) classical extrapolation. Darkest (black) lines show the total distribution, and color-coded lines show the inclusive distributions for certain energy and angular bins (described in the keys). 


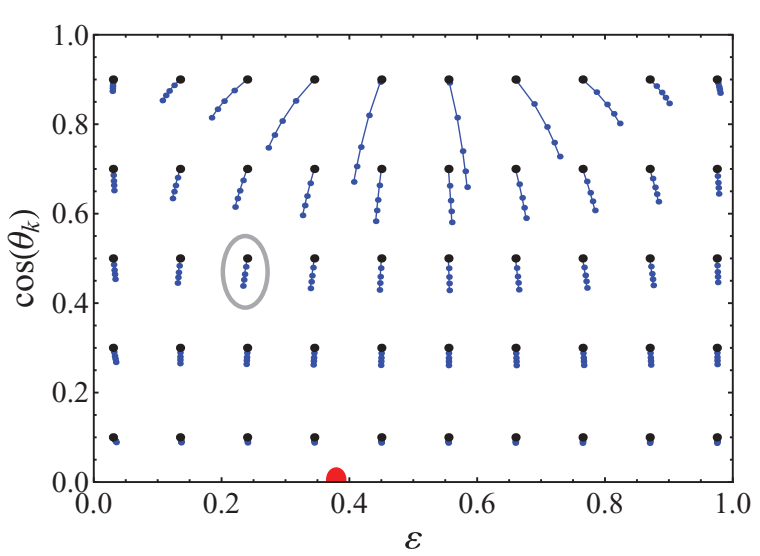

FIG. 12. (Color online) Classical trajectories on the kinematical plane $\left\{\varepsilon, \cos \left(\theta_{k}\right)\right\}$ for ${ }^{45} \mathrm{Fe}$ in the "T" Jacobi system, $E_{T}=1.154 \mathrm{MeV}$. Starting points (larger circles) correspond to $\rho_{\max }=1000 \mathrm{fm}$. Dots in the curves correspond to $\rho_{\mathrm{ext}}=1400,2200,4000$, and $10^{5} \mathrm{fm}$. The (red) dot on the $x$ axis, $\cos \left(\theta_{k}\right)=0$, corresponds to a stationary point; see the discussion in Sec. VIC.

The improvement of the momentum distributions owing to the $\mathrm{CE}$ for ${ }^{45} \mathrm{Fe}$ is demonstrated in Fig. 14 for the complete momentum distributions and in Fig. 15 for the inclusive ones. The most impressive modifications are for the $\varepsilon$ distribution in the "Y" system and for the $\cos \left(\theta_{k}\right)$ distribution in the " $T$ " system. Because these distributions have bell shapes, centered at (or close to) the center of the kinematical range, we can characterize them in terms of the full width at half-maximum (FWHM). CE decreases this value by about $30 \%$ for $\cos \left(\theta_{k}\right)$ in the "T" system and by about $10 \%$ for $\varepsilon$ in the "Y" system. This effect is sufficiently large to be already observable at the current level of the experimental precision.

The experimental distribution for ${ }^{45} \mathrm{Fe}$ [7] has quite low statistics (150 events) and therefore it is far from being smooth [see Fig. 16(a)]. To make a visual comparison with theoretical calculations possible, we produce a "smooth" representation of these data based on the experimental uncertainties. The raw experimental data measured in Ref. [7] by an optical time projection chamber consists of the energies and the polar angles of the two protons and the azimuthal angle between the projections of the two protons' momenta on the cathode
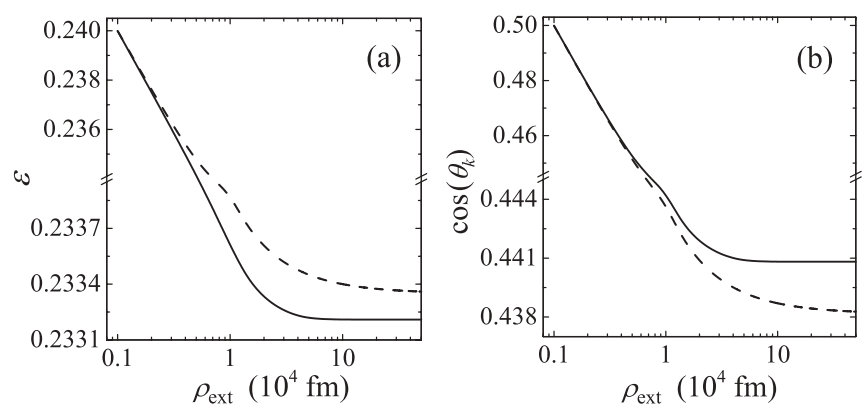

FIG. 13. Radial stabilization of the values $\varepsilon$ (a) and $\cos \left(\theta_{k}\right)$ (b) with $\rho$ in the case of screened Coulomb potential (solid curves) and in the case of nuclear Coulomb potential only (dashed curves) for one selected trajectory in ${ }^{45} \mathrm{Fe}$ (see Fig. 12). $E_{T}=1.154 \mathrm{MeV}$, $\rho_{\max }=1000 \mathrm{fm}$.
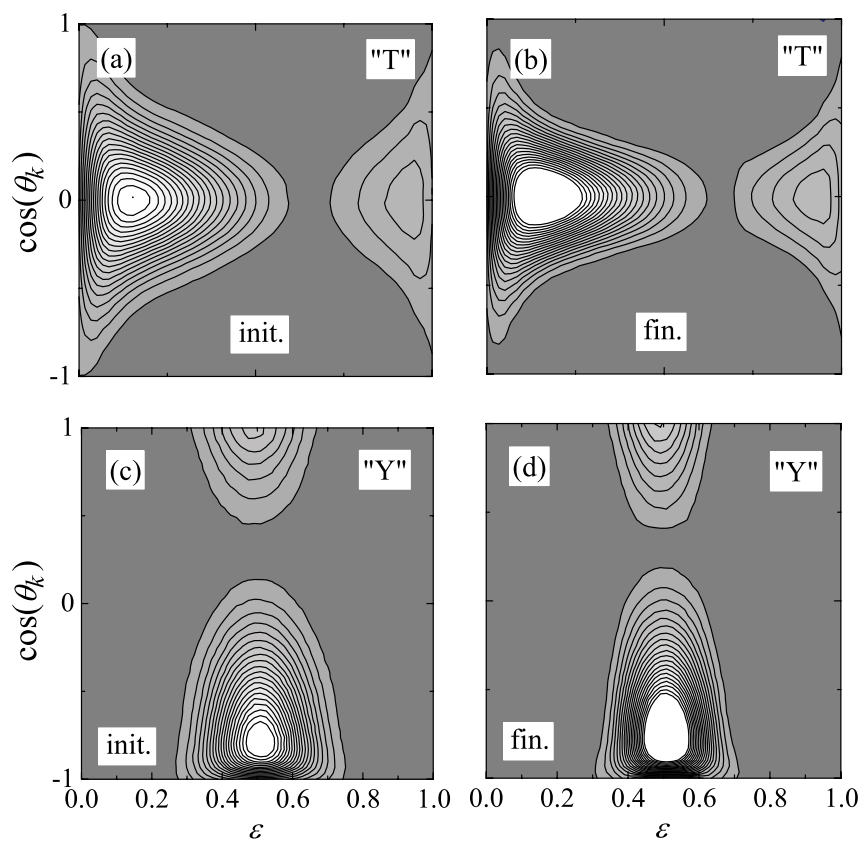

FIG. 14. Contour maps of the momentum density distribution on the kinematical plane $\left\{\varepsilon, \cos \left(\theta_{k}\right)\right\}$ for ${ }^{45} \mathrm{Fe}$ in the "T" (a, b) and "Y" (c, d) Jacobi coordinate systems without (a, c; "init.") and with (b, d; "fin.") classical extrapolation.

plane of the chamber. Each parameter for each event has a value (and its uncertainty) defined individually by a complex iterative fitting procedure. Instead of each event, we generate an event distribution based on the stochastic Gaussian variation
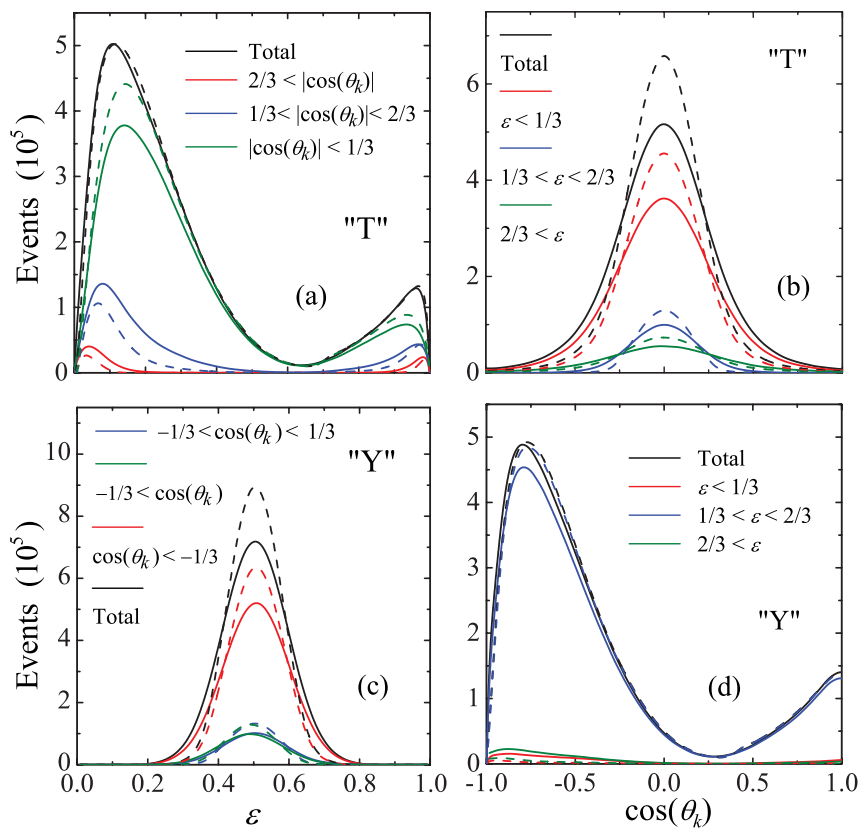

FIG. 15. (Color online) Inclusive energy and angular distributions for ${ }^{45} \mathrm{Fe}$ in the "T" (a, c) and "Y" (b, d) Jacobi coordinate systems without (solid curves) and with (dashed curves) classical extrapolation. Darkest (black) lines show the total distribution, and color-coded lines show the inclusive distributions for certain energy and angular bins (described in the keys). 

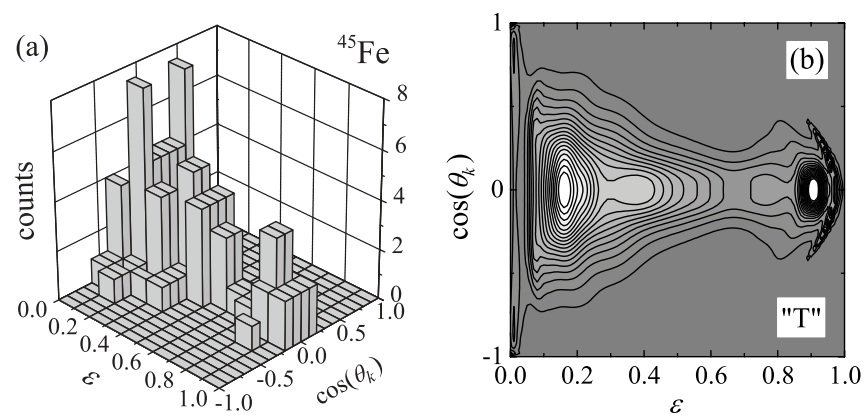

FIG. 16. Experimental momentum density distribution in ${ }^{45} \mathrm{Fe}$ in the " $T$ " Jacobi system. (a) Original distribution from Ref. [7] is shown as a histogram. (b) "Smooth" version of this distribution taking into account experimental errors is shown as a contour plot.

of each parameter within its uncertainty range. So instead of one point in the kinematic space we get a kind of a "probability cloud." The result of this procedure is shown in Fig. 16(b). This procedure is not a cure for small statistics, but for small statistics and large experimental uncertainties we think it is a preferable presentation, as it incorporates information about the distortions caused by the measurement procedure in a consistent and visible way.

Experimental data are compared with inclusive theoretical distributions sensitive to the CE in Fig. 17. In this plot,
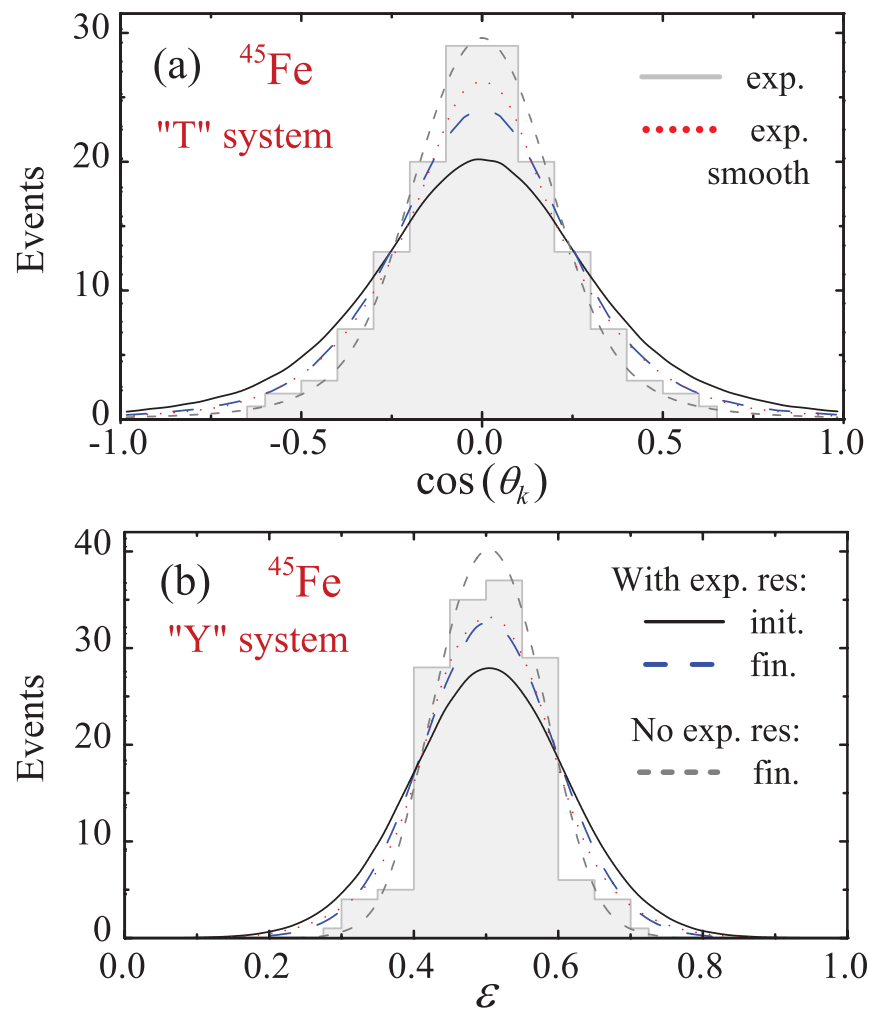

FIG. 17. (Color online) Inclusive angular (a) and energy (b) distributions for ${ }^{45} \mathrm{Fe}$ in the "T" and "Y" Jacobi coordinate systems without ("init.") and with ("fin.") classical extrapolation compared to the experimental data. Results with experimental resolution from Ref. [7] and without it are explained in the keys, which are the same for both plots. theoretical results were treated by the procedure that is closest to the experimental treatment of the data: (i) for the "theoretical event" the nearest experimental event in the space of parameters $\left\{E_{p 1}, E_{p 2}, \theta_{1}, \theta_{2},\left|\phi_{2}-\phi_{1}\right|\right\}$ was defined, (ii) spherical coordinates for protons from the theoretical event were distributed according to the errors of the nearest experimental event, (iii) the momentum of the core was reconstructed and the total energy of the "distorted" theoretical event was renormalized to correspond exactly to the experimental one, and (iv) a new location in the kinematical plane $\left\{\varepsilon, \cos \left(\theta_{k}\right)\right\}$ was defined. The effect of the experimental resolution is a roughly $25 \%$ increase in the FWHM for the $\varepsilon$ distribution and an $18 \%$ increase in the FWHM for the $\cos \left(\theta_{k}\right)$ distribution (see Fig. 17). It can also be seen in Fig. 17 that the theoretical results with $\mathrm{CE}$ are in quantitative agreement with the experiment, while without $\mathrm{CE}$ they are not completely consistent with the data. So we have appreciable experimental evidence that the long-range treatment of the momentum distributions (namely, $\mathrm{CE}$ ) is necessary for heavy $2 p$ emitters.

\section{DISCUSSION}

\section{A. Classical motion}

It is important to note that large hyper-radii are used to start the CE procedure. Specifically, for true $2 p$ decay with such large hyper-radii, practically the whole WF resides in the classically allowed region (the probability of finding the system in the classically forbidden region is very low). For example, for the ${ }^{45} \mathrm{Fe}$ calculation with hyper-radius $\rho_{\max }=$ $1000 \mathrm{fm}$ and MC generation of $10^{7}$ events, it is typical that not a single event is generated that is situated in the classically forbidden region. This fact confirms the validity of the choice of a hypersphere as the surface at which the switching from quantum-mechanical to classical methods is performed.

\section{B. Electron screening}

Discussion of the ${ }^{45} \mathrm{Fe}$ case can provide an illustrative example here. So far, the decay process of ${ }^{45} \mathrm{Fe}$ with a half-life of $2.6 \mathrm{~ms}$ [7] was measured in gas (or solid-state) detectors. This means that at the moment of decay, ${ }^{45} \mathrm{Fe}$ has completely recovered the electron shell. The Bohr radius for ${ }^{45} \mathrm{Fe}$ is

$$
a_{0}=\frac{1}{m_{e} \alpha Z}=2035 \mathrm{fm},
$$

where $Z=\sum_{i} Z_{i}$ is the total charge of ${ }^{45} \mathrm{Fe}$. Therefore, we can expect that the screening effect of the innermost electrons becomes observable at about $2000 \mathrm{fm}$. Classical trajectories for ${ }^{45} \mathrm{Fe}$ in kinematical space are well stabilized by $10^{5} \mathrm{fm}$, but there is a minor drift up to much larger distances. It is clear that some effect of the electron screening on the momentum distributions can be expected.

The binding energy of all electrons estimated as independent particles is $\sum_{i} m_{e}\left(Z / 2 n_{i} \alpha\right)^{2}\left(n_{i}\right.$ is the principal quantum number of the shell), which gives 52.3 and $47.8 \mathrm{keV}$ for ${ }^{45} \mathrm{Fe}$ and ${ }^{43} \mathrm{Cr}$, respectively. So when ${ }^{45} \mathrm{Fe}$ emits two protons, at least two electrons should be ejected, carrying away $4.5 \mathrm{keV}$ of energy. The estimated velocities of protons with energies around $0.5 \mathrm{MeV}$ and electrons with energies around $1 \mathrm{keV}$ are 

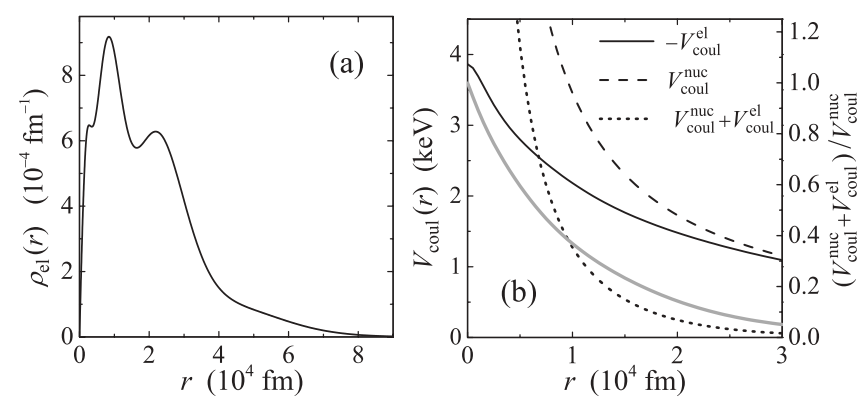

FIG. 18. (a) Electron density for the 24 lowest electron shells in ${ }^{45} \mathrm{Fe}$ (normalized for integration over $d r$ ). (b) The left axis shows the proton potential for Coulomb interaction of the nucleus, the electron shell, and their difference (screened potential). The ratio of the screened to the nuclear potential is shown by the solid light (gray) curve opposite the right axis.

0.033 and 0.063 , respectively. These velocities are comparable, which means that the $2 p$ decay of atomic ${ }^{45} \mathrm{Fe}$ would be accompanied by a strong reconstruction of atomic structures having the same time scale. It is reasonable therefore to make estimates of a screening with the ${ }^{45} \mathrm{Fe}$ electron density, but only for 24 electrons. This will somehow account for the effect of the electron shell disintegration during the $2 p$ decay of ${ }^{45} \mathrm{Fe}$ and provide a nuclear plus atomic Coulomb potential tending to 0 at infinity.

The electron densities used for the screening calculations and the potentials obtained are shown in Fig. 18. One can see already that at $2000 \mathrm{fm}$, the full $\left(V_{\text {coul }}^{\text {nuc }}+V_{\text {coul }}^{\text {el }}\right)$ Coulomb potential is noticeably reduced, owing to the screening, compared to the nuclear Coulomb potential (the reduction factor is 0.8 ). At $7000 \mathrm{fm}$ the reduction factor is 0.5 , and at $30000 \mathrm{fm}$ it tends to 0 .

The radial stabilization of the values $\varepsilon$ and $\cos \left(\theta_{k}\right)$ in the screening case compared to the purely nuclear case is shown in Fig. 13 for one selected trajectory. It can be seen that in the screening case, the trajectory stabilizes at $\rho \sim(3-4) \times 10^{4} \mathrm{fm}$. In the purely nuclear case, the minor drift of the trajectory continues to much larger $\rho$ values. The calculations show that in the " $\mathrm{T}$ " system, the screening effect is largest for the variable $\cos \left(\theta_{k}\right)$. It is typically at the level of $0.6 \%$ of the absolute value of this variable, and for $\rho_{\max }=1000 \mathrm{fm}$, it typically accounts for $3 \%-4 \%$ of the CE effect. For an effect that is $0.6 \%$ at the absolute scale, it is difficult to speculate about its observability just now: its scale is comparable to the widths of the lines in our plots. However, if we think about it as an effect of the atomic surroundings on nuclear decay properties, then such a value can be considered an impressive one.

It should be noted that the existence of the screening effects is the subject of the experimental technique employed. For example, the $2 p$ decay in ${ }^{19} \mathrm{Mg}$ was studied in the decay-in-flight experiment in Ref. [6]. In this experiment the ${ }^{19} \mathrm{Mg}$ g.s. was populated by the neutron knockout from the relativistic beam of the completely stripped ${ }^{20} \mathrm{Mg}$ ions. The resulting ${ }^{19} \mathrm{Mg}$ is also completely stripped and can hardly pick up any electrons before the decay. Therefore, despite the long lifetime $\left(T_{1 / 2}=4 \mathrm{ps}\right.$, which is much longer than the typical recombination time), screening in this experiment will have a different character compared to the case discussed previously for ${ }^{45} \mathrm{Fe}$.

\section{Self-similar solutions}

From Figs. 9 and 12, it is possible to see that there exist socalled "stationary points" in the kinematical $\left\{\varepsilon, \cos \left(\theta_{k}\right)\right\}$ plane in the " $\mathrm{T}$ " system. For such points, the classical trajectories in this plane have 0 length. For the degenerate situation $\varepsilon \equiv 1$, the stationary behavior is trivial; this situation is not very interesting, as the phase space for such configurations in the quantum-mechanical problem tends to 0 . However, there exist nondegenerate stationary points that, for a decay into two protons and a heavy core $\left\{A_{3}, Z_{3}\right\}$, are

$$
\varepsilon=\frac{\left(1+A_{3} / 2\right)\left(Z_{3} / A_{3}\right)^{2 / 3}}{2 A_{3}+\left(Z_{3} / A_{3}\right)^{2 / 3}}, \quad \cos \left(\theta_{k}\right)=0 .
$$

These stationary points are defined by the condition that the force acting on each particle is always directed exactly along the line connecting that particle with the center of mass of the whole three-body system. Such stationary points should exist for any two-body potential with the same power dependence on radius $V(r) \sim r^{n}$ for each pair of the particles. The values of $\varepsilon$ equal to $0.497,0.382$, and 0.379 are found for ${ }^{6} \mathrm{Be},{ }^{19} \mathrm{Mg}$, and ${ }^{45} \mathrm{Fe}$, respectively, by Eq. (25) as well as by a direct calculations using Eq. (6). It is clear that the solution, which is a stationary one in the $\left\{\varepsilon, \cos \left(\theta_{k}\right)\right\}$ plane, is an analog of the Lagrange solution in celestial mechanics (with the difference that we are dealing here with repulsive $1 / r$ potentials).

The multicluster decays of nuclear systems has been qualitatively studied in Ref. [20]. In this work a quasi-classical approach was used, based on the classical self-similar solutions of the few-body Coulomb problem. The stationary point discussed previously represents such a self-similar solution in our specific case. It was concluded in Ref. [20] that "three-cluster configuration asymptotically approaches to an expanding self-similar triangle whose sides obey the $(M / Z)^{1 / 3}$ rule." This statement is probably not completely correct. It can be seen from Figs. 9 and 12 that there is a trend for classical trajectories to tend somehow toward the stationary point that corresponds to a self-similar solution. This trend leads to certain systematic modifications of the momentum distributions by the long-range Coulomb interaction. However, as we have seen in this work, the whole picture is more complex. The total distributions occupy broad regions of the kinematical plane. They are determined mainly by the internal structure of the three-body system and the decay dynamics under the barrier than by the long-range Coulomb interaction outside of the barrier. In general, the classical trajectories started from a hypersphere of large radius converge to final positions that have nothing to do with stationary points.

\section{CONCLUSION}

In this work we discuss the extrapolation along the classical trajectories as a method to improve the momentum distributions for radioactive $2 p$ decay (true three-body decay). The proposed method provides a near-perfect description of the distributions in the test cases of simplified three-body 
Hamiltonians. In the case of real three-body Coulomb interactions, considerable quantitative effects on the distributions are observed. In the case of the lightest $2 p$ emitter, ${ }^{6} \mathrm{Be}$, this effect is minor, but in the heavier $2 p$ emitters $\left({ }^{19} \mathrm{Mg}\right.$ and $\left.{ }^{45} \mathrm{Fe}\right)$ the improvement is essential for precise description of the distributions.

It should be emphasized that some aspects of the momentum distributions for $2 p$ decays are sensitive to the long-range three-body Coulomb interaction, while others are absolutely insensitive. Namely, the angular distribution in the Jacobi " $\mathrm{T}$ " system and the energy distribution in the Jacobi "Y" system are considerably modified by the CE. Two other inclusive distributions (the energy distribution in the Jacobi " $T$ " system and the angular distribution in the Jacobi "Y" system) are essentially not influenced by the CE. Therefore the long-range part of the three-body Coulomb interaction does not practically change the information about the internal structure of the decaying system that is contained in the latter distributions.

Attention should be paid to the huge range that is required both for the extrapolation range $\left(\sim 10^{5} \mathrm{fm}\right)$ and for the starting point of the classical procedure $\left(\sim 10^{3} \mathrm{fm}\right)$ under typical decay conditions. The classical procedure is applicable only for distances above 500-1500 fm (for the $\rho$ variable) for the considered set of $2 p$ emitters (which is actually quite representative). The intermediate distances, from 30 to $100 \mathrm{fm}$ (where protons come from under the Coulomb barrier) to about $1000 \mathrm{fm}$, should to be treated quantum mechanically to obtain decent results from the $\mathrm{CE}$.

We have shown that electron screening can have a sizable effect on the momentum distribution in the $2 p$ decay of atomic ${ }^{45} \mathrm{Fe}$. So the $2 p$ radioactivity belongs to a rare class of nuclear phenomena that exist on the borderline with atomic phenomena. There are examples of weak radioactive decays modified by atomic electrons (e.g., owing to the energy conditions making $\beta^{-}$decay possible only into bound electron states [21] or because of the hyperfine effect [22]). We believe that the considerable sensitivity of the radioactive decay via particle emission owing to a modification of the potential barrier properties in the atomic environment is demonstrated in our work for the first time.

\section{ACKNOWLEDGMENTS}

L.V.G. acknowledges the support from Deutsche Forschungsgemeinschaft Grant No. 436 RUS 113/907/0-1, a FAIR-Russia Research Center grant, Russian Foundation for Basic Research Grant Nos. RFBR 08-02-00892 and RFBR 08-02-00089-a, and Russian Ministry of Industry and Science Grant No. NSh-7235.2010.2.
[1] V. I. Goldansky, Nucl. Phys. 19, 482 (1960).

[2] M. Pfützner et al., Eur. Phys. J. A 14, 279 (2002).

[3] J. Giovinazzo et al., Phys. Rev. Lett. 89, 102501 (2002).

[4] L. V. Grigorenko et al., Phys. Rev. C 80, 034602 (2009).

[5] I. Mukha et al., Phys. Rev. C 77, 061303(R) (2008).

[6] I. Mukha et al., Phys. Rev. Lett. 99, 182501 (2007).

[7] K. Miernik et al., Phys. Rev. Lett. 99, 192501 (2007).

[8] I. Mukha et al., Nature 439, 298 (2006).

[9] L. V. Grigorenko, R. C. Johnson, I. G. Mukha, I. J. Thompson, and M. V. Zhukov, Phys. Rev. Lett. 85, 22 (2000).

[10] L. V. Grigorenko, R. C. Johnson, I. G. Mukha, I. J. Thompson, and M. V. Zhukov, Phys. Rev. C 64, 054002 (2001).

[11] L. V. Grigorenko, and M. V. Zhukov, Phys. Rev. C 68, 054005 (2003).

[12] L. V. Grigorenko and M. V. Zhukov, Phys. Rev. C 76, 014008 (2007).
[13] L. V. Grigorenko and M. V. Zhukov, Phys. Rev. C 76, 014009 (2007).

[14] L. V. Grigorenko, I. G. Mukha, I. J. Thompson, and M. V. Zhukov, Phys. Rev. Lett. 88, 042502 (2002).

[15] L. V. Grigorenko, I. G. Mukha, and M. V. Zhukov, Nucl. Phys. A 713, 372 (2003); 740, 401(E) (2004).

[16] L. V. Grigorenko, I. G. Mukha, and M. V. Zhukov, Nucl. Phys. A 714, 425 (2003).

[17] L. V. Grigorenko et al., Phys. Lett. B 677, 30 (2009).

[18] L. Rosenberg, Phys. Rev. D 8, 1833 (1973).

[19] S. P. Merkuriev, Theor. Math. Phys. 32, 680 (1977).

[20] O. I. Kartavtsev, Few-Body Syst. 34, 39 (2004).

[21] M. Jung et al., Phys. Rev. Lett. 69, 2164 (1992).

[22] Yu. A. Litvinov et al., Phys. Rev. Lett. 99, 262501 (2007). 